This item was submitted to Loughborough's Research Repository by the author.

Items in Figshare are protected by copyright, with all rights reserved, unless otherwise indicated.

\title{
Service-aware multi-constrained routing protocol with QoS guarantee based on fuzzy logic
}

PLEASE CITE THE PUBLISHED VERSION

PUBLISHER

(c) IEEE

VERSION

VoR (Version of Record)

\section{LICENCE}

CC BY-NC-ND 4.0

\section{REPOSITORY RECORD}

Jing, Zuo, Chi Xuefen, Lin Guan, and Li Hongxia. 2019. "Service-aware Multi-constrained Routing Protocol with Qos Guarantee Based on Fuzzy Logic”. figshare. https://hdl.handle.net/2134/4057. 
This item was submitted to Loughborough's Institutional Repository (https://dspace.lboro.ac.uk/) by the author and is made available under the following Creative Commons Licence conditions.

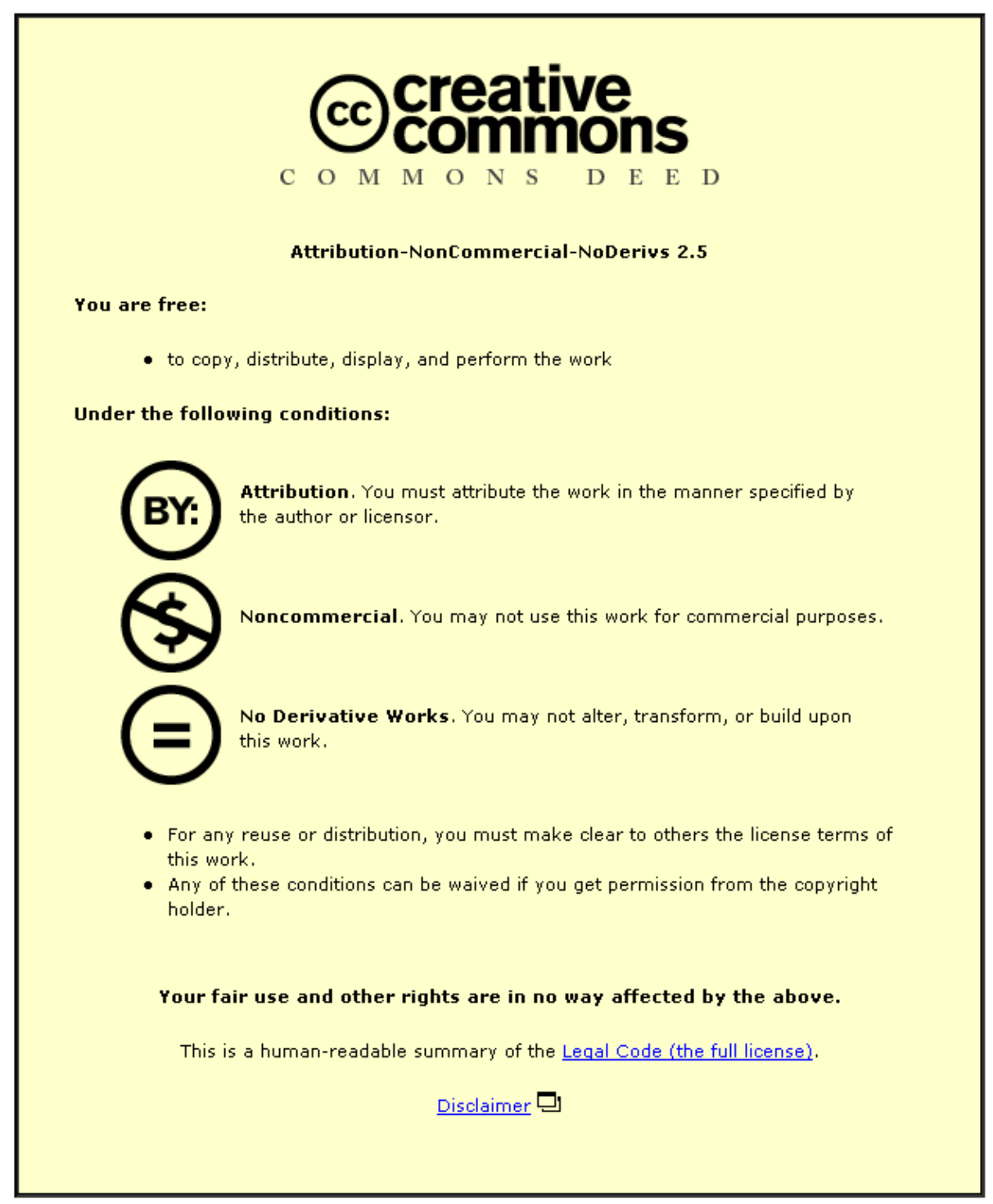

For the full text of this licence, please go to: http://creativecommons.org/licenses/by-nc-nd/2.5/ 


\title{
Service-aware Multi-constrained Routing Protocol with QoS Guarantee Based on Fuzzy Logic
}

\author{
Zuo Jing*, Chi Xuefen*, Lin Guan ${ }^{\dagger}$, Li Hongxia* \\ * School of Communication Engineering, Jilin University, China \\ ${ }^{\dagger}$ Department of Computer Science, Loughborough University, UK \\ chixf@jlu.edu.cn
}

\begin{abstract}
Single-constrained QoS (Quality of Service) routing protocols have inherent shortcomings when they are applied to ad hoc networks. Only considering a single constraint parameter can't always cope with the problems aroused by the uncertainty of ad hoc networks well. This paper proposes a multiconstrained QoS routing protocol based on fuzzy logic. The protocol is service-aware. It considers the QoS requirements asked by different kinds of services and takes different network state parameters as the constraint conditions. New route informing mechanism is proposed to support route update; speed of packets sending is adjusted in terms of the output of fuzzy system; and routing algorithm for real time traffic is optimized to assure that data are always transmitted through the route with the lowest delay. The simulation results show that the improved protocol has better features on delay, delay variation, packet loss rate and throughput compared to single-constrained QoS routing protocol.
\end{abstract}

\section{Introduction}

A mobile ad hoc network is a collection of wireless mobile nodes that form a temporary network without any established infrastructure or centralized authority [1]. With its special applications and advantages, ad hoc network is expected not only to provide data service but also to support other types of services, such as voice, image, video and even integrated services. So, improving and extending the capability of QoS guarantee in ad hoc networks has become one of the research hotspots in wireless communication field. Single-constrained routing protocols have received success in many aspects, however, they do not always perform best. In fact, the change of ad hoc network state connects with several factors, such as network topology, transmission range, available bandwidth and node energy. If all the constraint parameters are considered, changes of one parameter may not result in big change of network performance. However, selecting a route which satisfies all multiple constraints is an NP complete problem. There is no accurate mathematical model to describe it. Additionally, the transformation of dynamic topology causes the change of QoS parameters, and finally results in the uncertainty of QoS information. Fuzzy logic is a theory that not only supports several inputs, but also has good adaptability for imprecise information. So adopting fuzzy logic to solve the problems in ad hoc networks is an appropriate choice.

Many routing protocols based fuzzy logic have been proposed for ad hoc networks. A. Cohen presents a new fuzzy-based method for path selection. The goal of the path selection process is to identify a feasible path while minimizing the overall route setup time [2]. Hui Liu proposes a Genetic Fuzzy Multi-path Routing Protocol whose performance outperforms that of DSR, SMR and SBMR when their packet delivery ratio, endto-end delay and the frequency of route discovery are compared [3]. Susan Rea proposes a fuzzy logic based caching decision as a route selection method. This facilitates the generation of effective caches that limits the necessity for route discovery floods [4]. Xu Zhang proposes a fuzzy logic QoS dynamic source routing which can tolerate a high degree of information imprecision. The added fuzzy logic module integrates the QoS requirements of application and the routing parameters to determine the most qualified route [5].

As reviewed above, previous algorithms have something in common. They all employ the idea of fuzzy logic which is proved to be feasible in mobile ad hoc networks and achieve the target of satisfying QoS requirements. However, they don't differentiate the 
types of traffic. In this paper we explore a novel service-aware, multi-constrained QoS routing protocol based on fuzzy logic. In our routing protocol, aiming at providing different QoS guarantee to real time service and non real time service, two sets of fuzzy logic are developed. Constraint conditions used by two sets of fuzzy logic are gathered and renewed separately and dynamically to adapt to the varying QoS requirements of two kinds of traffic. Because route stability[6] affects network performance greatly, a novel scheme is proposed in this paper, in which fuzzy logic system is used to guarantee the stability of route.

\section{Fuzzy logic system modeling}

Using fuzzy logic system to analyze those dynamic systems which are too complex to be described by the accurate mathematical model is an important application of fuzzy theory. The characteristics of fuzzy logic happen to fit for the uncertainty of network parameters resulted from the dynamic features of ad hoc networks.

\subsection{Selection of constraint conditions}

This section discusses the constraints for different services employed by fuzzy system.

Number of hops is a parameter used most frequently in route selection. If data is transmitted through the route with the least hops, the possibility of route failure and packet loss is lower, and the route is more stable. In addition, the least hops mean the lowest delay from source to destination. Mobile speed of a node can also affect the stability of networks. The faster the mobile speed is, the more easily the nodes will move out of each other's transmission range, and the more possible the link failure is. If the link being used fails, route rediscovery will start, and network delay will increase. At the same time, packet loss will be more possible.

Real time traffic is very sensitive to delay and delay variation. One of the important factors for real time traffic is the bandwidth which affects the network delay. If bandwidth resource is insufficient, queuing delay will increase and congestion may appear. If some packets are discarded due to congestion, they need to be retransmitted, which results in even heavy delay. Non real time traffic is sensitive to packet loss rate. In ad hoc networks packet buffer occupancy rate is usually used to present the network congestion degree. When congestion degree deteriorates to a degree that network begins to discard packets, the network performance can't be tolerated by non real time traffic no longer, especially by FTP traffic. So we choose packet buffer occupancy rate to measure the packet loss rate of each path. Packet buffer occupancy rate for a node is denoted by $\frac{q_{i}}{b_{i}}, q_{i}$ is the length of all packets stored in buffer of node $\mathrm{i}$, and $b_{i}$ is the buffer capacity of node i. The packet buffer occupancy rate of the whole path is calculated by

Bufferccupancyrate $=\frac{\sum q_{i}}{\sum b_{i}}$

$\sum q_{i}$ is the sum of length of packet queue for all nodes on the path. $\sum b_{i}$ is the sum of packet buffer capacity for all nodes on the path.

\subsection{Fuzzy logic system design}

The structure of the proposed fuzzy logic system is shown in Figure 1.

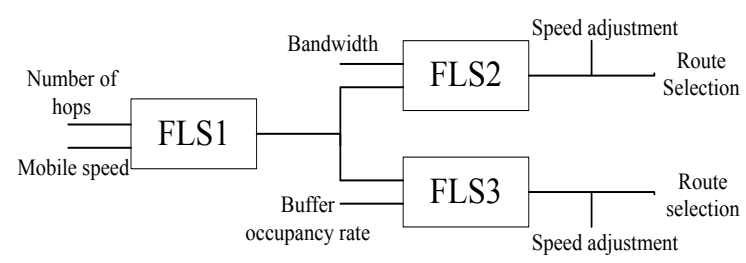

Figure 1. Fuzzy logic system model

The system consists of three subsystems. Subsystem1 (FLS1) is responsible for calculating route stability by fuzzy logic theory. Number of hops and mobile speed are the input parameters. The output is the route stability. Type of the traffic is examined before feeding the output result of FLS1 to the next fuzzy logic system. Real time flow is sent into FLS2 which is responsible for calculating the end-to-end delay of each route. Bandwidth and route stability are the input parameters of FLS2. According to the end-toend delay, which is the output of FLS2, the speed of sending packets is adjusted and the route with the lowest delay is chosen. If the flow is non real time flow, it will be sent into FLS3. The function of FLS3 is calculating the packet loss rate of each route. Its structure is similar to that of FLS2. Here the input parameters are packet buffer occupancy rate and route stability, the output is packet loss rate. According to the packet loss rate, the speed of sending packets is adjusted and the route with the lowest packet loss rate is selected. The fuzzy logic system operates in all source nodes.

Three major processes are designed in fuzzy logic system. They are fuzzification, fuzzy inference and defuzzification. 
2.2.1. Fuzzification and selection of membership functions. According to network scales, node configurations and QoS requirements of different traffic, input parameters and output parameters are respectively described using the following linguistic variables. For input parameters, the terms "short", "medium" and "long" are used to describe number of hops, the terms "slow", "medium" and "fast" are used to describe mobile speed. The terms used to describe bandwidth and packet buffer occupancy rate are "low", "medium" and "high". For output parameters, route stability is described using "low", "medium" and "high", end-to-end delay and packet loss rate are described using 5 linguistic variables: "very low", "low", "medium", "high" and "very high".

Although the selection of membership functions relates to expert knowledge which is widely subjective, there are still some basic principles to obey. It should be noted that selection of membership functions affects the sensitivity of the fuzzy logic system greatly [7]. With the advantages of simplicity and high computational efficiency, triangular functions have been extensively used in fuzzy logic system of ad hoc networks. In this paper, triangular function is taken as the membership function of fuzzy system. Here we only present the membership functions of number of hops (scale of 0-10hops) and end-to-end delay (scale of 05s). They are shown in Figure 2 and Figure 3. Vertical coordinates represents the degree of membership, which distributes in the interval of $[0,1]$. The membership functions of other input parameters and output parameters are similar to Figure 2 and Figure 3.

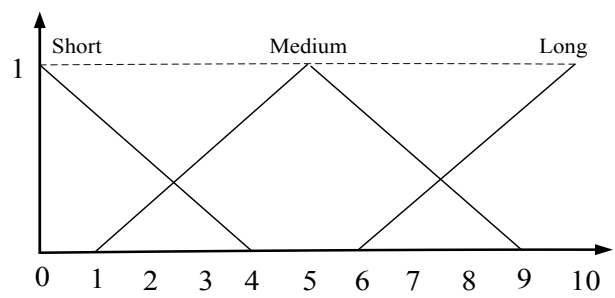

Figure 2. Membership function of number of hops

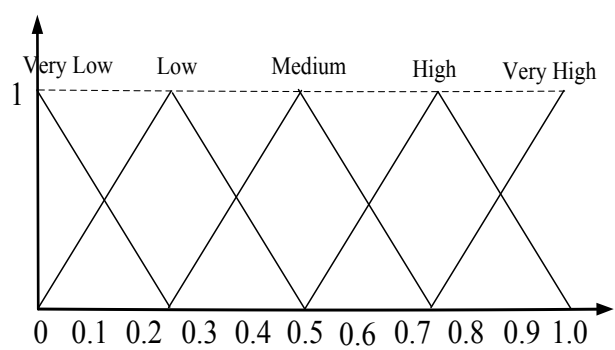

Figure 3. Membership function of end-to-end delay
2.2.2. Fuzzy inference rules. The design of fuzzy inference rules is based on the analysis of the experimental results. For FLS1, mobile speed has higher weighing than number of hops, for FLS2, bandwidth is more important than route stability; and for FLS3, packet buffer occupancy rate is more important than route stability. Figure 4, Figure 5 and Figure 6 show the detailed inference rules used in our fuzzy logic system. The rules are realized in the form of "IF-THEN". The input parameters are combined using "and".

\begin{tabular}{|c|c|c|c|}
\hline \multirow{3}{*}{ 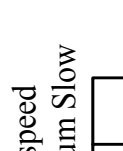 } & \multicolumn{3}{|c|}{ Number of Hops } \\
\hline & Short & Medium & Long \\
\hline & High & High & High \\
\hline & High & Medium & Medium \\
\hline & Medium & Low & Low \\
\hline
\end{tabular}

Figure 4. Fuzzy rules of FLS1

\begin{tabular}{|c|c|c|c|}
\hline \multirow{3}{*}{ 至 } & \multicolumn{3}{|c|}{ Route Stability } \\
\hline & Low & Medium & High \\
\hline & Very High & High & Medium \\
\hline & High & Medium & Low \\
\hline & Medium & Low & Very Low \\
\hline
\end{tabular}

Figure 5. Fuzzy rules of FLS2

\begin{tabular}{|c|c|c|c|}
\hline \multirow{5}{*}{ 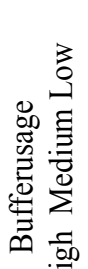 } & \multicolumn{3}{|c|}{ Route Stability } \\
\hline & Low & Medium & High \\
\hline & Medium & Low & Very Low \\
\hline & High & Medium & Low \\
\hline & Very High & High & Medium \\
\hline
\end{tabular}

Figure 6. Fuzzy rules of FLS3

2.2.3. Defuzzification. Defuzzification is selecting an appropriate method to calculate a representative precise value from a fuzzy set. This paper adopts weighted mean method, which is one of the most popular defuzzification methods in fuzzy logic control system. The formula is

$$
z^{*}=\sum \mu_{c}(\bar{z}) \cdot \bar{z} / \sum \mu_{c}(\bar{z})
$$

Where $\bar{Z}$ represents the centroid of each output membership function, $\mu_{c}(\bar{z})$ indicates the strength of the output membership function, and $z^{*}$ is the crisp value output from defuzzifier. 


\section{Multi-constrained routing protocol with QoS guarantee based on fuzzy logic}

Multi-constrained routing protocol with QoS guarantee based on fuzzy logic is implemented as an extension and optimization to the DSR (Dynamic Source Routing). The goal of optimized protocol is to find out the best route which meets specified QoS requirements of different kinds of traffic. For collecting arguments demanded by fuzzy system, route discovery process of DSR is optimized; RREQ (route request packet) and RREP (route reply packet) are modified. According to the output of fuzzy logic system, we adjust the speed of sending packets at source node to make protocol more self-adapted. We optimize protocol algorithms for real time traffic to make sure that data is always transmitted through the route with the lowest delay.

\subsection{Route discovery and speed adjustment}

In multi-constrained routing protocol, all constraint parameters are designed to be collected and transferred by route discovery process. RREQ and RREP are extended with three additional fields: Bandwidth lptr, Queue_len_lptr and Buffer_capacity_lptr, as shown in Figure 7. They are used to store bandwidth, length of packet queue and buffer capacity of each node.

Bandwidth_lptr $\quad$ Queue_len_lptr $\quad$ Buffer_capacity_lptr

\section{Figure 7. New added fields to the head of RREQ and RREP}

Before source node sends packets, it first examines its route cache to check the routes to the destination. If routes are found, fuzzy logic system is started. It completes fuzzy computation for every route and makes routing decisions. If no route is found, route discovery is initiated. The source node broadcasts a RREQ to its neighbors. During the process of route discovery, bandwidth, length of packet queue and buffer size of each node are gathered and stored in RREQ. When RREQ arrived at the destination or an intermediate node which has active routes to the destination, a RREP is created and sent back to source node, by which source node is informed that a new route to the destination has been discovered. At the same time, bandwidth and packet buffer occupancy rate of the whole path are obtained by calculating the gathered parameters.

Also, we should find the relationship between the current sending speed and the initial sending speed before packets are sent. So we refer to the outputs of
FLS2 and FLS3, which are delay and packet loss rate separately, to adjust the speed of sending packets. Table 1 presents the speed adjustment scheme for real time traffic in detail. Same plan is also adopted by non real time traffic, the only change is that delay is replaced by packet loss rate.

Table 1. Speed adjustment of real time traffic

\begin{tabular}{|c|c|c|c|}
\hline $\begin{array}{c}\text { Initial } \\
\text { speed }\end{array}$ & $\begin{array}{c}\text { Current } \\
\text { speed }\end{array}$ & $\begin{array}{c}\text { Level of } \\
\text { delay }\end{array}$ & $\begin{array}{c}\text { Speed after } \\
\text { adjustment }\end{array}$ \\
\hline $\mathrm{V}$ & $\mathrm{V}$ & High & $(1 / 2) \mathrm{V}$ \\
\hline $\mathrm{V}$ & $\mathrm{V}$ & Very High & $(1 / 4) \mathrm{V}$ \\
\hline $\mathrm{V}$ & $(1 / 2) \mathrm{V}$ & Medium & $\mathrm{V}$ \\
\hline $\mathrm{V}$ & $(1 / 2) \mathrm{V}$ & Very High & $(1 / 4) \mathrm{V}$ \\
\hline $\mathrm{V}$ & $(1 / 4) \mathrm{V}$ & Medium & $\mathrm{V}$ \\
\hline $\mathrm{V}$ & $(1 / 4) \mathrm{V}$ & High & $(1 / 2) \mathrm{V}$ \\
\hline
\end{tabular}

\subsection{Protocol optimization of real time traffic}

In source-initiated on-demand routing protocols, the information in route table of each node can't be updated in time when network state changes. For real time traffic, the delay of an active route keeps changing, which may be different from delay calculated by fuzzy logic. If all the packets of a session always follow the originally selected route, QoS requirements may not always be met. Aiming at solving this problem, we propose a new delay informing mechanism, in which the change of end-to-end delay is informed by a new RINF (route inform packet). Its main fields are shown in Figure 8. The first three fields are as same as that of RREQ and RREP. Field updated delay presents the delay of the route being used.

We extend the source packet with additional 5 fields which is shown in Figure 9. Field suboptimal delay indicates the delay of second best route to the same destination existing in route cache. Field sending time is the time when the first data packet is sent. The delay informing mechanism includes three steps. The first step, selecting the best route based on fuzzy logic system and inserting suboptimal delay into the data packet. The second step, calculating the current route delay when data packets arrive at destination node. And the third step, informing source node and updating delay if current route delay is higher than suboptimal delay. After that the following packets will be sent along the new route which is selected based on renewed data. The mechanism makes sure that data packets are always sent by the route with the lowest delay.

\begin{tabular}{|l|l|l|l|}
\hline $\begin{array}{l}\text { Bandwidth } \\
\text { lptr }\end{array}$ & $\begin{array}{l}\text { Queue_len } \\
\text { lptr }\end{array}$ & $\begin{array}{l}\text { Buffer_capacity } \\
\text { lptr }\end{array}$ & $\begin{array}{l}\text { Updated } \\
\text { delay }\end{array}$ \\
\hline
\end{tabular}

Figure 8. New added fields to the head of RINF 


\begin{tabular}{|c|l|l|l|l|}
\hline $\begin{array}{c}\text { Band } \\
\text { width } \\
\text { lptr }\end{array}$ & $\begin{array}{l}\text { Queue } \\
\text { _len_lptr }\end{array}$ & $\begin{array}{l}\text { Buffer } \\
\text { capacity } \\
\text { lptr }\end{array}$ & $\begin{array}{l}\text { Suboptimal } \\
\text { delay }\end{array}$ & $\begin{array}{l}\text { Sending } \\
\text { time }\end{array}$ \\
\hline
\end{tabular}

Figure 9. New added fields to the head of source packet

Fuzzy Parameters of bandwidth, length of packet queue and packet buffer capacity are carried by RREQ, RREP, source route packet, acknowledgement packet and RINF. Any node when it receives one kind of these packets will update the parameters and store them in its own route cache. All these optimizations make the protocols more adaptable for the quickly changed network state.

\section{Simulation and results}

In this simulation, 25 mobile nodes are randomly placed in a rectangular field of 5000 meters by 5000 meters. Each node has a radio propagation range of 1000 meters. Two kinds of traffic, video conferencing and FTP, are configured. The domain of each input and output parameters are defined as follows.

Number of hops: [0,10hops];

Mobile speed: [0, $5 \mathrm{~m} / \mathrm{s}]$;

Bandwidth: $[0,1 \mathrm{Mb} / \mathrm{s}]$;

Packet buffer occupancy rate: [0,100\%];

Route stability: $[0,100 \%]$;

End-to-end delay: $[0,5 \mathrm{~s}]$;

Packet loss rate: $[0,100 \%]$.

We conduct experiments to evaluate and compare the performance of DSR and multi-constrained routing protocol with QoS guarantee based on fuzzy logic. The mobile speed is $1 \mathrm{~m} / \mathrm{s}$ and simulation time is 5 minutes for each session. Simulations are based OPNET. Some figures can't be depicted directly in OPNET, so, for keeping all figures in the same format, we collect data and use common drawing tool to depict all figures in this paper.

For real time traffic, we evaluate the features of endto-end delay and delay variation which are measured in the application layer. Figure 10 shows the comparison of end-to-end delay between the improved protocol and DSR. As the Figure 10 shows, our improved protocol has lower delay compared to DSR. Figure 11 shows the delay variations of two protocols. It is clear that our optimized protocol presents lower delay variation after 3 minutes and the delay variation changes more smoothly. Feature of delay variation is more important than feature of delay for real time services. People can bear delay but can't accept jitter of delay. So our improved protocol can provide better QoS to end user. For non real time traffic, we analyze the performance of the packet loss rate. Packet loss rate of a session is the ratio of the number of packets that are dropped by the network over the number of packets submitted to the network by the FTP source [8]. From Figure 12, we can see that the improved protocol has lower packet loss rate than DSR. Network throughput is depicted in Figure 13 which shows that the throughput of the improved protocols is higher than that of DSR.

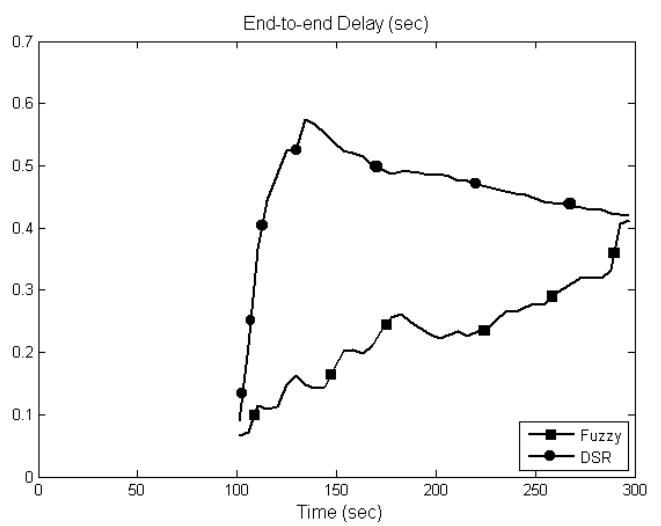

Figure 10. Comparison of end-to-end delay

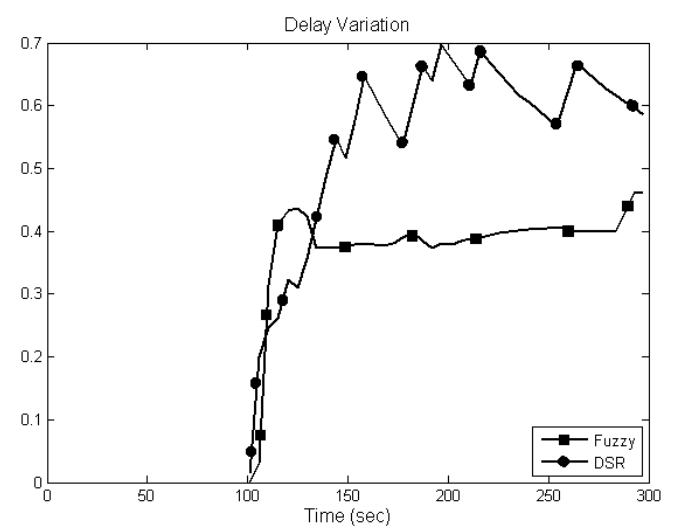

Figure 11. Comparison of delay variation

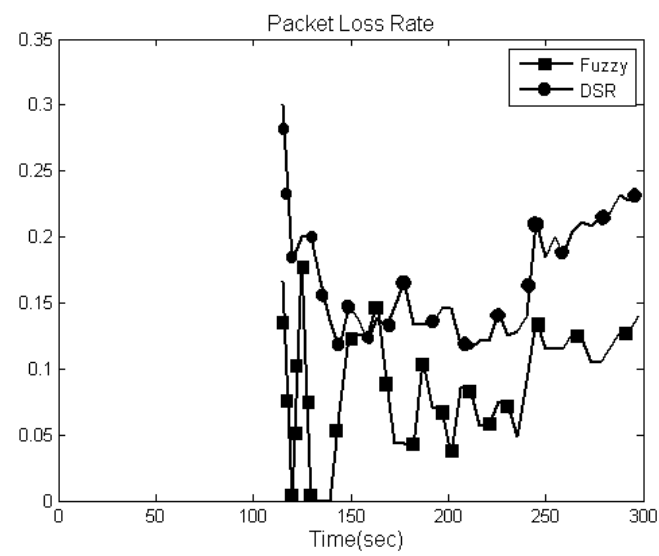

Figure 12. Comparison of packet loss rate 


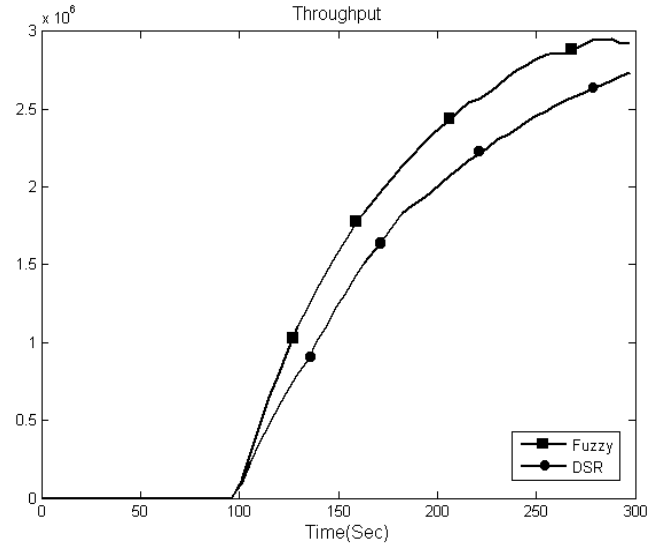

Figure 13. Comparison of throughput

All the results of simulations demonstrate that our optimized protocol outperforms the conventional DSR protocol. If only one single constraint condition is considered when making route decision, for instance, as DSR, QoS can't be guaranteed, because the constraint condition may changes during a session and other factors can also affect network performance. Our improved protocols always consider several constraint parameters. The changes of one or two parameters can't affect system functions seriously. Our route informing mechanism assures that routing data is renewed in time. Benefiting from all these optimizations, the improved protocol is more adaptable to the dynamic network than the conventional DSR.

\section{Conclusions}

Multi-constrained routing protocol with QoS guarantee based on fuzzy logic is proposed in this paper, it is developed based on DSR. Accounting for the uncertainty of route information in ad hoc networks, fuzzy logic system is adopted. The proposed protocol is service-aware in that different constraints are considered and different fuzzy logical systems are adopted according to the QoS demands asked by different kinds of traffic. It represents better traffic pertinence, which is hardly achieved by other ad hoc routing algorithms at present. Simulation results illustrate that the improved protocol has better performance than conventional single-constrained protocol for both real time and non real time services.

\section{Acknowledgments}

This research was supported by Department of Science and Technology of Jilin Province (20060525).

\section{References}

[1] S. Alampalayam and A. Kumar, "An Adaptive and Predictive Security Model for Mobile Ad Hoc Networks", Wireless Personal Communications, Kluwer Academic Publishers, Vol. 29, June 2004, pp. 263-281.

[2] A. Cohen, E. Korach, M. Last and R. Ohayon, "A Fuzzybased Path Ordering Algorithm for QoS Routing in Nondeterministic Communication Networks", Fuzzy Sets and Systems, Elsevier, Vol. 150(3), Mar 16 2005, pp. 401-417.

[3] H. Liu, J. Li, Y.Q. Zhang and Y. Pan, "An Adaptive Genetic Fuzzy Multi-path Routing Protocol for Wireless AdHoc Networks", Proceedings - Sixth Int. Conf. on Softw. Eng., Artificial Intelligence, Netw. and Parallel/Distributed Computing and First ACIS Int. Workshop on SelfAssembling Wireless Netw., SNPD/SAWN 2005, Institute of Electrical and Electronics Engineers Computer Society, Vol. 2005, May 23-25 2005, pp. 468-475.

[4] S. Rea and D. Pesch, "Multi-Metric Routing Decisions for Ad Hoc Networks using Fuzzy Logic", 1st International Symposium on Wireless Communication Systems 2004, Sep 20-22 2004, pp. 403-407.

[5] X. Zhang, S. Cheng, M.Y. Feng and W. Ding, "Fuzzy Logic QoS Dynamic Source Routing for Mobile Ad Hoc Networks", The Fourth International Conference on Computer and Information Technology, 2004, Institute of Electrical and Electronics Engineers Computer Society, Sep 14-16 2004, pp. 652-657.

[6] B. An and S. Papavassiliou, "An Entropy-Based Model for Supporting and Evaluating Route Stability in Mobile Ad Hoc Wireless Networks", IEEE Communications Letters, Institute of Electrical and Electronics Engineers Inc., Vol. 6(8), August 2002, pp. 328-330.

[7] J. Nie, J. C. Wen, J. Luo, X. He and Z. Zhou, "An Adaptive Fuzzy Logic Based Secure Routing Protocol in Mobile Ad Hoc Networks", Fuzzy Sets and Systems, Elsevier, Vol. 157(12), June 16 2006, pp. 1704-1712.

[8] C. Venkatesh, N. Yadaiah and A. M. Natarajan, "Dynamic Source Routing Protocol Using Fuzzy Logic Concepts for Ad Hoc Networks", Academic Open Internet Journal,http://www.acadjournal.com/2005/v15/part6/p5/DS R_fuzzy.pdf, Vol.15, 2005 